\title{
Demonstration of STEM Holography Using Diffraction Gratings
}

Benjamin J. McMorran ${ }^{1}$, Tyler R. Harvey ${ }^{1,2}$, Colin Ophus², Jordan Pierce ${ }^{1}$, Fehmi Yasin ${ }^{2}$

1. Department of Physics, University of Oregon, Eugene, Oregon, USA.

2. IV. Physical Institute, University of Göttingen, Göttingen, Germany.

3. National Center for Electron Microscopy, Molecular Foundry, Lawrence Berkeley National Laboratory, Berkeley, California, USA.

We are developing a new technique for STEM holography (STEMH), a scanning interferometric technique for generating phase contrast. However, where pioneering efforts in the 80s and 90s [1-3] used a biprism to produce two probes, we demonstrate the use of fast imaging detectors and a pre-specimen nanoscale grating as a beam splitter that can produce more than two probes [4]. In STEM mode, the multiple focused probes are separated by 10 s of nanometers in the specimen plane. One of the diffracted probes passes through a specimen, while the other diffracted orders pass through vacuum. The postspecimen imaging optics of the TEM then defocus and overlap the probes, forming an interference pattern at the imaging detector (Figure 1). Careful alignment of the descan and a selected area aperture allows us to record the interference pattern from only two beams.

We show preliminary experimental results in which we scan the beams across an amorphous carbon test specimen, recording an image of the interference pattern at each probe position. STEMH and HAADF signals can be recorded simultaneously, and the thickness reported by HAADF can be used to predict the evolution of the interference pattern (Fig. 2 top row). 2D images were also collected using a fast electron camera (Fig. 2 bottom row). We developed theory to process the 4D-STEM interference data, and it can also work on datasets involving interference between more than two beams. In principle, this analysis can be performed in real time during a scan. The advent of fast direct electron imaging detectors makes STEM holography practical [5].

\section{References:}

[1] J. M. Cowley, Ultramicroscopy 96 (2003), p. 163.

[2] M. Ichikawa, Y. Yajima, M. Takeshita, and T. Kobayashi, "Scanning interference electron microscopy," U.S. patent US5298747 A (March 29, 1994).

[3] T. Leuthner, H. Lichte, and K.-H. Herrmann, Phys. Stat. Sol. (a) 116 (1989), p. 113.

[4] F. S. Yasin et al, Microsc. Microanal. 22 (2016), p. 506.

[5] Work at the Molecular Foundry was supported by the Office of Science, Office of Basic Energy Sciences, of the U.S. Department of Energy under Contract No. DE-AC02-05CH11231. Work at University of Oregon was supported by the U.S. DOE under Award No. DE-SC0010466. This material is based upon work supported by the National Science Foundation Graduate Research Fellowship Program under Grant No. 1309047. 

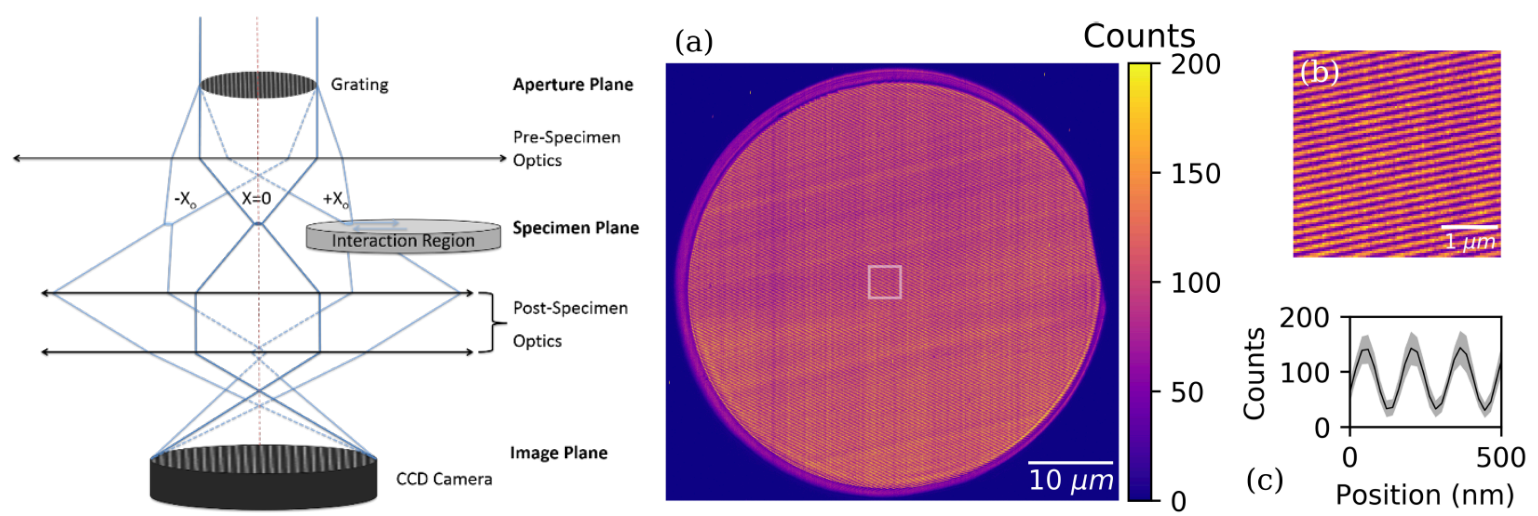

Figure 1. (Left) Schematic of a grating-based electron interferometer in a TEM. A single grating placed in the condenser aperture of a STEM creates multiple probes at the specimen plane (center image) separated by $50 \mathrm{~nm}$ or more. One of the diffracted probes passes through a specimen and acquires a phase.
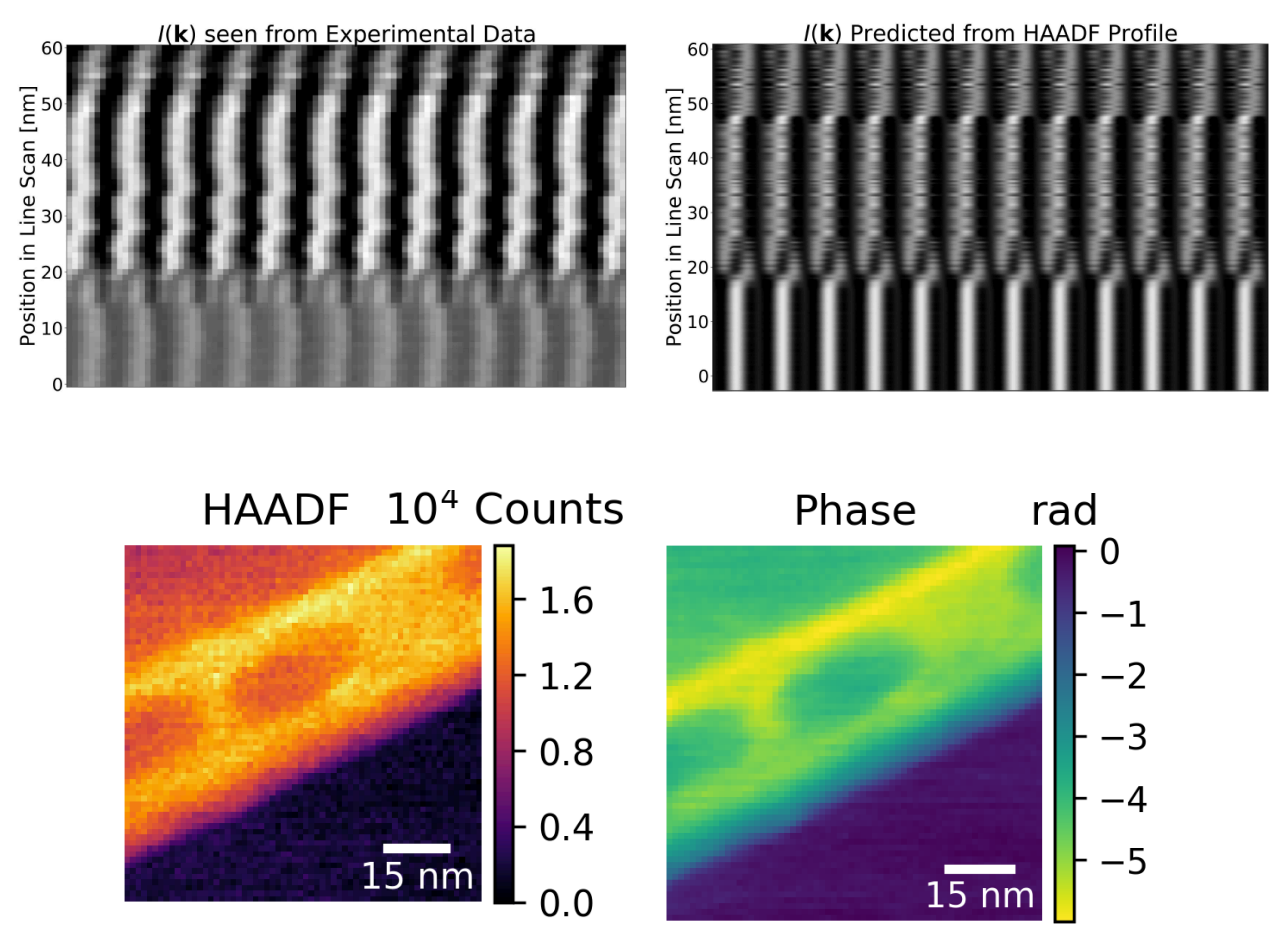

Figure 2. STEMH and HAADF measurements can be recorded simultaneously. Multiple diffracted probes are scanned across an amorphous carbon sample while their interference pattern and a HAADF signal are recorded at each probe position. (Top left) The evolution of interference pattern as one beam is scanned across a sample can be predicted from the sample thickness estimated by the HAADF signal and a simple optical model. (Top right) The experimental multiple-beam interference pattern evolves as predicted. 2D STEMH scans of lacey carbon produce a phase contrast image (lower right) which agrees with the mass-thickness contrast shown in the HAADF scan (lower left). 